\title{
A Gene Network Model for Developing Cell Lineages
}

\author{
Nicholas Geard ${ }^{1 *}$ and Janet Wiles ${ }^{1,2}$ \\ ${ }^{1}$ School of Information Technology and Electrical Engineering \\ ${ }^{2}$ School of Psychology \\ The University of Queensland, \\ St Lucia, Queensland 4072, Australia \\ Telephone: +61733651636 \\ \{nic, j.wiles\}@itee.uq.edu.au
}

\begin{abstract}
Biological development is a remarkably complex process. A single cell, in an appropriate environment, contains sufficient information to generate a variety of differentiated cell types, whose spatial and temporal dynamics interact to form detailed morphological patterns. While several different physical and chemical processes play an important role in the development of an organism, the locus of control is the cell's gene regulatory network.

We designed dynamic recurrent gene network (DRGN) model and evaluated its ability to control the developmental trajectories of cells during embryogenesis. Three tasks were developed to evaluate the model, inspired by cell lineage specification in C. elegans, describing the variation

${ }^{*}$ Corresponding author.
\end{abstract}


in gene activity required for early cell diversification, combinatorial control of cell lineages and cell lineage termination. Three corresponding sets of simulations compared performance on the tasks for different gene network sizes, demonstrating the ability of DRGNs to perform the tasks with minimal external input. The model and task definition represent a new means of linking the fundamental properties of genetic networks with the topology of the cell lineages whose development they control.

Keywords: gene regulation, cell lineage, development, recurrent network, C. elegans, DRGN 


\section{Introduction}

The development of a single, fertilised egg cell into a multicellular organism is one of the most complex processes in biology [38]. Each cell in a growing embryo contains an identical set of instructions encoded in its genome, yet it follows a unique developmental trajectory that specifies both the type of cell into which it will differentiate, as well as its location in the embryo. One major area of investigation within developmental biology is how the developmental trajectories of cells are programmed by a distributed network of interacting genes [7].

The field of artificial life has made extensive use of the concept of development, which it recognises as a powerful and highly evolvable means of encoding design solutions [3]. While this view has led many researchers in artificial life to approach biology as a source of novel ideas capable of revolutionising engineering and other domains [32], artificial life also has the potential to make a

contribution to theoretical biology [9]. Two requirements render this a challenging task; suitable computational models must be chosen to represent biological systems, and these models must be communicated in a fashion that is accessible to a wider scientific audience.

A requirement for modelling any complex system is to determine an appropriate level of abstraction, at which the properties that the model seeks to investigate will emerge from interactions between well-understood agents at a lower level of description. The goal when developing models of biological systems is to balance simplicity with plausibility. If a model is too simplistic, extensions to biology may be unconvincing, and findings will lack cogency. Conversely, overly elaborate models that include unnecessary details will obscure the essential dynamics of the process under investigation. Furthermore, to communicate to a biological audience, the computational tasks in a simulation study need to address identifiable aspects of biological phenomena. 
Development is an interesting computational challenge because the information required to specify each of a potentially large number of cell lineages is contained in the regulatory interactions of a single network of genes. A variety of genetic regulatory network (GRN) models have been developed to simulate aspects of biological development, including the effect of interplay between multiple interacting mechanisms [12, 16], the role played by physical interactions [10] and the dynamics of intercellular communication [13]. One issue that has not been explicitly addressed is the relationship between the regulatory complexity of a GRN and its ability to autonomously specify the developmental trajectories of a group of cells.

In this study, we developed a dynamic recurrent gene network (DRGN $\left.{ }^{1}\right)$ model, capable of generating developmental cell lineages. Our hypothesis is that this model, despite its simplicity, possesses a sufficiently flexible range of dynamic behaviours to enable it to generate complex developmental trajectories. Specifically, this study considers the situation in which a GRN receives only limited external input and so is almost exclusively reliant on its internal regulatory interactions to store a developmental program.

The next section of this paper reviews the biological background of our study, briefly describing the process of development and the role that gene regulation plays in determining a cell's fate. Previous computational approaches to modelling gene regulation and development are then reviewed before the DRGN model is introduced. Several tasks are used to explore the capabilities of the model, including the initial diversification of cells, the generation of specific patterns of differentiation and the control of cell lineage termination. Finally, the performance of the model and potential implications of the results discussed.

\footnotetext{
${ }^{1}$ For ease of pronunciation, we refer to these networks as DRaGoNs.
} 


\section{Development and Cell Lineage}

The development of an embryo involves a variety of interacting processes, including the growth, division and differentiation of cells and the specification of body plans. Although the formation of physical structure in a developing embryo involves mechanical factors such growth, cell migration and differential adhesion, the information that determines whether the end product will be a worm or a whale is encoded in the genome [7]. This section describes the process of cell differentiation and the role that gene regulation plays in specifying cell lineages.

\subsection{Cell Differentiation}

Cell differentiation is the process by which cells undergo physical and chemical changes that result in them becoming structurally and functionally distinct. From an initial egg cell, each successive cell division results in a new generation of cells whose final fate is more determined. Upon reaching its terminal, fully differentiated state, each cell will function as, for example, a nerve, muscle or blood cell.

The primary feature that determines the function of a fully differentiated cell is the proteins it contains [1]. Similarly, the most important property characterising a developing cell is its pattern of gene activity [38]. Externally, the cells of an early embryo may be virtually indistinguishable. Already, however, the differences in their patterns of gene activity determine the role they and their progeny will play in the fully developed organism. When a cell divides, this pattern of active and inactive genes is passed on to its daughter cells via positive regulatory feedback, and physical and chemical modifications to the genome [38].

Patterns of gene activity are dynamic, changing over time as cells become more differentiated. These changes are both a product of complex internal 
dynamics and a response to signals from the extracellular environment. The sequence of changes in gene expression that a cell exhibits on its way to becoming differentiated forms the developmental history, or lineage, of that particular cell. An organism's cell lineage is the sum of the lineages of its individual cells. Cell lineages form a binary tree structure, where the root node represents an initial egg cell, a branch point represents a division event and the leaf nodes represent a set of terminally differentiated cells. An important question in developmental biology is how the sequence of differentiation events is controlled to produce complex patterns of terminal cells.

\subsection{The Control of Cell Lineages by Gene Regulation}

In a eukaryotic cell, the genetic regulatory system is encoded in the genome, which is located in the cell's nucleus. As well as carrying out various functions related to cell maintenance and function, a significant portion of the genetic system is involved in programming embryonic development [38].

The process of gene expression begins when an RNA polymerase molecule binds to the start site of a gene, unwinds a section of DNA and uses one of the strands as a template to transcribe messenger RNA (mRNA) molecules. mRNA molecules are transported outside of the cell nucleus to the cytoplasm, where they are translated into proteins. Proteins can either be structural, enabling a cell to fulfil its functional role in an organism, or they can re-enter the nucleus to regulate the expression of other genes. These regulatory proteins, known as transcription factors (TFs), interact with the promoter and control regions of a gene to either enhance or inhibit the transcription of that gene. Some TFs are required for any transcription to occur at all. Others play a role as activators, binding to enhancer sites located upstream or downstream of the gene to facilitate transcription. Yet another type acts as a repressor, either by blocking activator TFs, or by preventing the binding of RNA polymerase to a 
gene start site [1].

When a cell divides, the set of TFs that determine its pattern of gene activation are divided between the daughter cells, so each will generally have a similar pattern of gene expression to its parent. On occasion, the distribution of TFs in the parent cell may be asymmetric [19]. The two daughter cells will therefore inherit different sets of regulatory information and hence follow two unique developmental trajectories.

Inductive signals originating from other cells or the environment can also alter patterns of gene expression. In general, these signalling molecules bind to receptors found on the cell surface, and the signal is transmitted to the nucleus via a series of chemical events called a signal transduction pathway. The role of these signals is selective, they choose one fate from among the relatively small number of possible trajectories defined by the current state of the cell [38].

For this study, the selection of an appropriate level of detail from the myriad of facts known about biological development required focusing on two essential aspects of gene regulation. Firstly, genes may be grouped into two classes, regulatory genes that control the expression of other genes (including other regulatory genes) and structural genes that specify the functional roles of different types of cells. Secondly, when a cell divides, both daughter cells inherit the genetic network of the parent cell, including the structure of the regulatory interactions and their level of activity. However, they also receive information that differentiates between them, either through asymmetric division of determinants or inductive signals from other cells.

\subsection{The Embryonic Development of C. elegans}

Designing appropriate tasks to test a model of development requires explicitly defining the genetic information inherent in control decisions. Gene expression microarrays are one possible source of gene expression patterns. However, mi- 
croarray data represents an average across a population of cells and therefore lacks the specificity of individual cell trajectories. Analysis of the phenotypic effects resulting from mutations to particular genes is another potential source of patterns, however, comprehensive data has been obtained for only a subset of the relevant genes [29]. Our approach therefore has been to examine the cell lineages themselves, with the aim of modelling the control decisions guiding differentiation as represented by the topology of the cell lineage and the identities of the terminal cells [6].

In most multicellular organisms, cell lineages follow a general plan for a given species, with a certain amount of variation in the trajectories of individual cells. Some species are remarkably resilient to environmental factors, with well established developmental trajectories. Others, such as many plant species, are very responsive to their environmental conditions. In this study, in order to target the control decisions for differentiation as closely as possible to a known biological system, we based the simulation tasks on the embryonic development of Caenorhabditis elegans (C. elegans), an organism with an exceptionally well characterised cell lineage.

C. elegans is a small (approximately $1 \mathrm{~mm}$ as an adult) worm found throughout the world. It has become an important model organism for developmental biologists for several reasons, some of which also make it an attractive subject for computational modelling. Most notably, it has a relatively small number of cells (959, plus a variable number of germ cells, as a developed adult) and an invariant cell lineage, making it possible to gather a considerable amount of data on its developmental processes. Despite its relative simplicity however, it shares many genetic mechanisms with other species, including humans [38].

The first complete observation of $C$. elegans embryogenesis was carried out in 1983 [36], resulting in a diagram of the entire cell lineage. Since then, there has been a gradual growth in understanding the way that gene expression and cellular interactions guide the developmental process, and while some important 
mechanisms have been uncovered, the full picture is still incomplete [24].

A significant advance in understanding $C$. elegans development occurred when new experimental techniques allowed the observation, not only of cell lineage, but also of cell position throughout embryogenesis [31]. Based on these observations, the three-dimensional structure of the initial stages of C. elegans embryogenesis was modelled computationally by Kitano and colleagues [22]. Their primary focus was on visualising cell position data, however, and only limited connections were made to the underlying dynamics of gene expression. By contrast, our model does not explicitly represent the physical structure of the embryo, but focuses on the sequence of control decisions underlying the developmental process, from which cell identity and embryonic structure emerge.

\section{Modelling Gene Regulation and Development}

Recent years have seen a rapid growth in the use of modelling as a tool to develop intuitions about biological processes, providing insights into natural computation for both artificial and real life researchers. Approaches to modelling gene networks range from detailed, mathematical models tied closely to experimental data (see $[8,15]$ for recent reviews) to more abstract models from the fields of complex systems and artificial life (e.g. [21, 34]). While the former is often used to make quantitative predictions and generate empirically testable hypotheses, the latter aims to develop insights about high-level properties of gene regulation.

The DRGN model follows the latter approach. It was inspired by biology, and aims to generate insights that can inform our understanding of biology. However, we use a level of description that attempts to capture the general principles of biological development while abstracting away from as much specific detail as possible. 


\subsection{Boolean and Continuous Models of Gene Regulation}

As described in Section 2.2, gene expression is regulated by interactions between the multiple transcription factors that bind to a gene's control region. Because many of these factors are the products of other gene transcription events, they too are under regulatory control. A genetic system can therefore be described in terms of a network in which nodes represent genes and links represent regulatory interactions. The state of a system at any given time can be described by the levels of activation of all the genes in the system. The activation of any given gene can be defined as a function of the current state of the system and any environmental inputs. Thus future states of the system can be predicted from the current pattern of gene expression.

Several different approaches have been applied to modelling gene regulation. In the simplest case, genes are represented as nodes that are either on or off (i.e. either expressed or not) and the condition for activation of a node is represented as a Boolean function of its input [21]. Depending on size, connectivity and the choice of node activation functions, such systems are capable of displaying a variety of dynamic behaviours, ranging from ordered fixed-point and cyclic attractors to disordered or "chaotic" dynamics [21, 39]. As well as being subjected to considerable theoretical investigation, Boolean networks have also been used to model specific biological processes such as the cell cycle [18] and pattern formation [4]. Other GRN models have used networks in which nodes have continuous, rather than Boolean, levels of activation [27, 33, 37]. Genes in biological systems display a continuous range of activity levels and it has been argued that using a continuous representation captures several properties of gene regulatory networks not present in the Boolean model [37]. For example, genes may have different effects depending on their level of expression that a simple on/off distinction does not allow. Also, a single gene may influence the transcription of different genes by differing amounts. 


\subsection{Using Gene Regulation as a Basis for Development}

Within the field of artificial life, developmental processes are recognised as an important ingredient in the artificial evolution of highly complex systems (for a recent review, see [35]). Development enables complex phenotypes to be represented in a more compact genotypic form by allowing modular components to be reused in different contexts. In addition, a suitable developmental process can potentially enable the generation of viable phenotypes in a range of different environmental conditions. Approaches to modelling development include both the use of generative grammars (e.g. [17]), in which a set of rules is recursively applied to simulate growth, and genetic models (e.g. [5]), in which pattern formation and growth processes emerge from GRN dynamics.

Several studies have focused on the way in which a GRN interacts with other processes to control development, including cell communication via diffusible signals $[12,23]$, intercellular interaction $[12,13,16]$, and constraints due to physical laws [10]. The approaches used in these studies differ in their strengths and limitations as models of biological systems. The representations used in some (e.g. [12]) are quite complex and, while capable of displaying an impressive range of behaviours, they are not readily amenable to the use of machine learning techniques to generate specific target behaviours. The model used by Bongard [5] for the co-evolution of agent morphologies and controllers is similarly expressive. However, the basic morphological units used in his model were macro-cellular, involving assemblies of sensors, actuators and neural control elements.

Hogeweg's model [16] also generates convincing simulations of a diverse range of morphological processes (e.g. gastrulation and limb budding), and uses a standard Boolean network for control. We have also chosen to use a relatively simple network description in this study, however, the behaviours we are investigating are at a lower phenomenological level. Where many of the studies 
mentioned focus on the morphological characteristics of development, we concentrate on the issue of controlling cell fate and this focus imposes a number of constraints on the definition of both the model and the evaluation tasks.

\subsection{Design constraints on a GRN model for cell lineage development}

This study involved the design and evaluation of a model of gene regulation capable of generating developmental trajectories. Key decisions in the study included the constraints on designing an appropriate set of tasks and the constraints on the mechanisms incorporated into the GRN model. In both cases, the goal was to abstract away from specific biological detail, while retaining relevant control principles.

Constraints on the design of appropriate tasks included:

- Cell lineages, at the level of abstraction chosen, are viewed as patterns of gene activity, with a single genetic network being responsible for generating the diversity of expression patterns throughout the developmental trajectories constituting the lineage of an organism.

- Both similarity and diversity between cell lineages need to be incorporated into the tasks, requiring the model to deal with the combinatorial nature of the division and differentiation task.

- The tasks must involve the generation of temporal patterns of expression to correctly specify the timing of critical cell differentiation decisions.

- The tasks must reflect aspects of a well-characterised biological organism to ground the study in biological phenomena of interest, requiring the model to cope with the complexities inherent in biological development, but not inventing tasks with complexities that are not grounded in biology.

Constraints on the design of the model included: 
- Gene interactions are viewed as a network of interacting controls, which allow multiple interactions between genes over the time periods characterising cell division.

- The model should be as simple as possible in order to focus on the relevant phenomena. It should be the simplest design that incorporates the biological mechanisms of interest and has the computational power to control the tasks specified, with the potential to explore its capabilities and limitations at depth.

- The model should be extensible, in the sense that it can be progressively elaborated to incorporate additional details of the known biology of genetic regulation and address tasks with increasingly detailed requirements.

\section{Methodology}

In line with the design constraints listed in the previous section, the basic requirements therefore are explicit control over the complexity of the task, the regulatory power of the network and the level of external input. The current study evaluated the extent to which a dynamic recurrent network, similar to a widely studied class of artificial neural network models known as recurrent neural networks [11], fulfils these requirements.

An advantage of a recurrent network representation is that it enables the model to express a complex range of gene interactions whilst generalising away from the specific biological processes that underly those interactions. Therefore, it is possible to start with a relatively "minimalist" model and fully explore its capabilities and limitations. With this knowledge as a baseline, future studies can extend the model to capture additional biological detail and the impact of these extensions can be properly assessed.

In order to evaluate the ability of our model to generate complex cell lin- 
eages, three tasks were designed, each corresponding to a particular aspect of the developmental process. The three processes selected were the rapid initial diversification of an undifferentiated egg cell into several distinct lineage branches, the use of combinatorial gene expression to specify unique lineages and the termination of division after cell lineage has been fully specified. While none of these alone is sufficient as a complete description of developmental control, they are all necessary components.

\subsection{The Recurrent Network Model}

In the DRGN model, a genetic system is defined as a network of $N$ interacting nodes (see Figure 1). The activation state of each node is a continuous variable in the range $[0,1]$, where 0 represented a completely inactive gene and 1 a fully expressed gene. The network is updated synchronously in discrete time steps, where each step represents the duration between cell divisions.

The network contains $N_{s}$ structural nodes, $N_{r}$ regulatory nodes and a single input node. The structural nodes represent a subset of genes whose pattern of activation specifies the current state of differentiation of the cell. These nodes have no regulatory outputs, that is, their level of expression has no influence on the future dynamics of the network. The regulatory nodes in the network represent genes that play a regulatory role only.

The sharp distinction between structural and regulatory roles for genes reflects the traditional definition of a gene as the region of DNA encoding a single protein [1]. Proteins are typically classed as either structural or regulatory and genes have traditionally been classified according to the type of protein they encode. It is now understood that the regulation of gene expression is a significantly more complicated process than initially thought [28]. Genes may code for multiple products via alternative splicing, and regulation may occur at stages other than transcription, such as RNA editing and translation control. In this 
respect therefore, our model represents a first approximation to the complexity of the regulatory process, with the potential for future refinement.

The input node was used to specify the relative position of the cell in the lineage. After division, this node was set to 0 in the left daughter and 1 in the right daughter. This minimal external input reflects the combined effects of the different contextual signals received by the two cells resulting from their respective positions in the embryo. A clear example of this type of signal is the pop-1 gene in C. elegans, which is differentially expressed in the two daughters produced following an anterior-posterior cell division [25]. It is important to note that, at the level of abstraction of the DRGN model, this input does not need to be assigned any single biological function. Rather than explicitly requiring that cell fate be specified by any particular mechanism (such as asymmetric division or inductive signals), it simply indicates that there is some difference in regulatory context between the two daughter cells.

In most organisms, the time-scale for a single cell division is measured in minutes or hours [1]. The time taken for an individual transcription event is generally shorter, therefore a single cell cycle may consist of multiple transcription events. To capture the potential complexity of the interacting transcription factors, we have used a network in which the regulatory nodes are fully connected. Thus an individual link in the network does not necessarily represent a direct physical interaction, but rather the degree of influence that the transcription of the source gene at time $t$ has on the transcription of the target gene at time $t+1$, where each time step represents a single cell division that may entail multiple transcription events.

These interactions can be summarised in a weight matrix, in which the entry at row $i$, column $j$ specifies the influence that gene $j$ has on gene $i$. These entries may be positive or negative, depending on whether the product produced by gene $j$ is an activator or a repressor in the regulatory context of gene $i$. A zero entry indicates that there is no interaction between the two genes. The inclusion 
of self-connections (i.e. from node $i$ to node $i$ ) allows for the possibility of genes influencing their own regulation.

The state of the network was updated synchronously, with the activation of node $i$ at time $t+1, a_{i}(t+1)$, given by

$$
a_{i}(t+1)=\sigma\left(\omega_{i} I(t)+\sum_{j=1}^{N_{r}} w_{i j} a_{j}(t)-\theta_{i}\right)
$$

where $N_{r}$ is the number of regulatory nodes, $\omega_{i}$ is the level of interaction from the single input, $I$, to node $i, w_{i j}$ is the level of the interaction from node $j$ to node $i, \theta_{i}$ is the activation threshold of node $i$, and $\sigma($.$) is the sigmoid function,$ given by

$$
\sigma(x)=\frac{1}{1+e^{-x}}
$$

\subsection{The Developmental Process}

The set of nodes and links of a DRGN represent the set of genes and their interactions in a model organism. To simulate the developmental process of the organism, the network was used to generate a cell lineage in the following way. The original network, representing a fertilised egg cell, was initialised by setting all node activations to 0 and the relative position input to 0.5 . The activation of each gene was then updated once. Cell division was implemented by creating two copies of the network with identical weights and node activations. The relative position input was set to 0 in the left daughter and 1 in the right daughter, representing the different contextual signals received by each cell as a consequence of its position in the embryo. The state of the network was again updated and a new division occurred. This process was repeated until the halting criterion of the particular task (described in Section 5) was met. 


\subsection{The Evolutionary Process}

To derive a suitable set of gene interactions for performing the cell lineage tasks, an automated machine learning technique was used to find a set of weights for each network. An evolutionary algorithm (EA) was selected as an appropriate search technique based on prior experience, the distributed nature of the representation being evolved and its extensibility for a variety of tasks and parameter settings. Note that, although EAs are inspired by evolutionary processes in biology, the intention in this study is not to model the evolution of $C$. elegans per se. Other automated learning techniques could also have been applied.

A simple evolutionary search strategy called the $1+1$ ES was used [2]. Initially a single network was generated with uniformly distributed random weights in the range $[-1,1]$. The error values for this individual were calculated as described below and stored. A new network was derived from this network by adding uniformly distributed random values in the range $[-0.5,0.5]$ to a randomly chosen subset of the network weights (typically 10\%). The error values for the modified individual were calculated and compared to that of the original individual. The individual with the lowest error was retained and used as the basis for the creation of a further new network. This process was repeated for a specified number of generations, or until a satisfactory solution was discovered (described in Section 5).

Two related error values were calculated for each target cell, measuring the difference between the pattern of activation of its structural nodes and the pattern of activation of the corresponding cell in the target lineage. One error value was based on the number of incorrect gene activations, the other incorporated a measure of the degree of incorrectness.

The first error value, the Number of Gene Errors (NGE) measured the number of incorrect structural gene activations in a cell. A correct gene activation was defined as being greater than 0.5 if the target activation was 1 , and less 
than or equal to 0.5 if the target activation was 0 . The NGE was calculated by

$$
N G E=\sum_{j=1}^{C} \sum_{i=1}^{N_{s}} \phi\left(p_{i}^{j}, f_{i}^{j}\right)
$$

where $C$ is the number of terminal cells in the lineage, $N_{s}$ is the number of genes in each target pattern, $p_{i}^{j}$ is the activation of gene $i$ in the target pattern $j$ and $f_{i}^{j}$ is the activation of the structural gene $i$ in the network corresponding to cell $j$ and $\phi($.$) is given by$

$$
\phi\left(p_{i}^{j}, f_{i}^{j}\right)= \begin{cases}0 & \text { if }\left(\left(p_{i}^{j}=1\right) \wedge\left(f_{i}^{j}>0.5\right)\right) \vee\left(\left(p_{i}^{j}=0\right) \wedge\left(f_{i}^{j} \leq 0.5\right)\right) \\ 1 & \text { otherwise. }\end{cases}
$$

The NGE was used in the first and third tasks to halt evolution when a "perfect" solution (no remaining incorrect gene activations) was found.

The second measure, the Sum Squared Error (SSE), measured the difference between the target activation (either 1 or 0 ) and the continuous-valued activation of each node. The SSE was calculated by

$$
S S E=\sum_{j=1}^{C} \sum_{i=1}^{N_{s}}\left(p_{i}^{j}-f_{i}^{j}\right)^{2}
$$

The SSE was used as the basis for comparing two networks during evolution.

\section{Cell Lineage Tasks}

\subsection{Task A: Initial Cell Diversification}

\subsubsection{Aim}

The initial cell divisions of $C$. elegans are invariant, with each precursor cell dividing to produce a somatic cell, which goes on to form part of the body of the embryo, and a further precursor cell. After the fourth division, the precursor cell forms the basis of the organism's germ line [38] (see Figure 2). The aim of the first task was to demonstrate how a DRGN could be used to simulate aspects 
of a well-characterised biological developmental sequence, and investigate its behavior by requiring it to generate a diverse set of expression patterns.

\subsubsection{Task Description}

The first task involved generating a cell lineage corresponding to the initial diversification of six founder cells in C. elegans embryogenesis, from which the remaining cells will develop.

In C. elegans (and many other organisms) there is not a clear relationship between the initial branches of the cell lineage and terminal cell fate. While the intestine and germ cells are all derived from the $\mathrm{E}$ and $\mathrm{P}_{4}$ cells respectively, epidermal cells are derived from both the $\mathrm{AB}$ and $\mathrm{C}$ cells. Conversely, the daughters of the $\mathrm{AB}$ cell will eventually differentiate into neuronal, epidermal and muscle cells [36]. Therefore, no strong correlations between the cells produced during initial diversification can necessarily be assumed. For this reason, we defined six random patterns of structural gene activation corresponding to the six cells at the beginning of each simulation trial (see Figure 2). The use of random patterns represents the lower limit of task correlation where each target developmental lineage is essentially arbitrary. Performance on a more correlated target was evaluated in Task B (see Section 5.2).

\subsubsection{Method}

In Task A, a lineage was developed until six cells corresponding to the AB, MS, E, C, D and $\mathrm{P}_{4}$ cells had been generated. The patterns of activation of the $N_{s}$ structural genes in each of these cells were then compared to the corresponding target pattern and the NGE and SSE calculated.

In order to explore the relationship between the task complexity and level of regulation required, a family of parameterised networks were generated, varying the size of the target pattern of each terminal cell $\left(N_{s}=4,8,12,16,20\right)$ and the number of regulatory genes $\left(N_{r}=2,4,8,16\right)$. For each structural-regulatory 
combination, 10 sets of target patterns and 10 initial networks were randomly generated. A set of target patterns was generated by initialising the target pattern of activation of each of the six cells such that each gene had a $50 \%$ chance of being on or off. Each network was tested against each target pattern (i.e. a total of 100 trials for each combination). Each trial was run for up to 50,000 generations, halting earlier if a solution with an NGE of zero was found.

For the purpose of comparison, a family of parameterised Boolean networks were also generated, varying the number of regulatory nodes $\left(N_{r}=4,8,16\right)$ and the level of connectivity $(K=2,4,6)$. These networks were evolved to match the same sets of target patterns as the DRGN model. In the case of the Boolean networks, the relative position input was incorporated by adding an additional node to the $N_{r}$ regulatory nodes that could have regulatory outputs to other nodes, but no regulatory inputs. Similarly, $N_{s}$ additional structural nodes were added that could have inputs from the regulatory nodes, but no outputs. A cell lineage was generated from the network using the same process as the DRGN (described in Section 4.2). The EA used to search for suitable networks was the same as that used for the DRGN (described in Section 4.3), except that, at each step, a new network was created using two steps. First, each regulatory interaction had was randomly rewired with probability 0.05 . Second, the output of each of the Boolean updating rules for a given node was flipped with probability 0.05 .

\subsubsection{Results}

The DRGNs demonstrated competence on the cell diversification task, with the majority of trials evolving a solution with greater than $90 \%$ accuracy (see Figure 5a). The best performance on all sizes was shown by networks with eight regulatory nodes (100\% for 4 -bit targets to $98.7 \%$ for 20 -bit targets). The networks with four and sixteen regulatory nodes achieved a similar level of accuracy. The performance of the network with only two regulatory nodes was 
considerably lower, suggesting that some minimum level of regulatory complexity was required to in order to perform the differentiation task. Interestingly, there appeared to be an "optimal" level of regulation. The networks with eight regulatory nodes consistently achieved higher levels of accuracy than those with sixteen regulatory nodes within the range of target patterns used in this study. A possible explanation is that this level of regulation represents a balance in the trade off between the complexity of the task and the complexity of the search space, which increases with the number of regulatory nodes. Furthermore, the length of time required for evolution to find a good network with eight regulatory nodes scaled more slowly as the size of the task increased (see Figure 5b).

While the performance of the network with two regulatory nodes was significantly less accurate, considering the size of the more complex tasks and the minimal input provided, the performance (over $80 \%$ accuracy) was still surprisingly good: twenty structural genes in each of six target patterns equates to a total of 120 bits of output, generated from only a single bit of input provided for each of five divisions. Clearly a significant amount of information can be stored by the regulatory interactions within a DRGN.

Only the results of the best performing set of Boolean networks $(\mathrm{N}=16 ; \mathrm{K}=$ 2) are shown (see Figure 5a). As can be clearly seen, all of the DRGNs (including those with two regulatory nodes) outperformed the Boolean networks. Closer investigation of the Boolean network solutions revealed that they were generally unable to make sufficient use of the input bit to differentiate their subsequent patterns of activation. The errors generally occurred when two sister cells (e.g. E and MS) remained on the same trajectory and hence displayed identical patterns of activation.

To provide insights into the space of possible DRGNs that the EA is searching, we calculated both the average performance of each individual network across the ten target patterns as well as the average performance on a particular target pattern across the ten networks. Each network on a particular target 
pattern tended to find solutions with similar levels of residual error, suggesting that good solutions could be reached by the EA from any part of the space. However, a greater level of variation was observed between the average residual error for different target pattern sets. Variation across the pattern sets indicates that some sets of patterns are consistently more difficult than others for the class of DRGNs. A likely cause is that the "easier" target pattern sets contain a higher degree of systematic structure, with fewer inconsistent interactions.

\subsection{Task B: Combinatorial Control of Cell Lineages}

\subsubsection{Aim}

A common feature of eukaryotic cell lineages is the use of combinatorial gene expression to allow a relatively large number of individual cell fates to be specified by a much smaller number of regulatory genes [1] (see Figure 3). In $C$. elegans, it has been proposed that specific genes may act as a binary switch to control the diversification of cell fates of a series of divisions [20]. The aim of the second task was to explore the ability of the DRGN model to generate complex, correlated developmental trajectories using combinatorial activation of a limited number of genes.

\subsubsection{Task Description}

After each cell division, the network was required to distinguish between the two newly created cells by the deactivation of a structural gene in the left daughter, and the activation of the corresponding structural gene in the right daughter. The target patterns of gene activation after the first division therefore, were "OFF" in the first gene of the left daughter and "ON" in the first gene of the right daughter. After the second division, "OFF-OFF" in the leftmost daughter, followed by "OFF-ON", "ON-OFF", and finally "ON-ON" in the rightmost daughter (see Figure 3). In an effort to prevent the EA from finding 
a solution capable of generating a single cell lineage to a great depth at the expense of all other lineages, a requirement was imposed to correctly match the target patterns of activation in all cells at a given level before the next level was generated (i.e. the two cells at the first level had to be correctly specified before the four cells at the second level were generated, and so on).

\subsubsection{Method}

The network structure, error measures and EA were used as described in Section 4. Again, the effect of different levels of regulation was explored by the use of a parameterised family of networks in which the size of the regulatory layer was varied $\left(N_{r}=2,4,8,16\right)$. Ten trials, each initialised with a random set of network weights, were run for each value of $N_{r}$. As the target pattern was open-ended (arbitrarily large cell lineages could potentially be generated), each trial has halted when there had been no further improvement in SSE for 50,000 generations.

\subsubsection{Results}

The largest combinatorial cell lineages were, unsurprisingly, generated by the networks with eight and sixteen regulatory nodes (see Figure 6). For each of these regulatory layer sizes, the EA managed to find at least one solution capable of generating the first five levels of cell division (32 terminal cells) with 100\% accuracy and the sixth level of cell division (64 terminal cells) with $87 \%$ accuracy (on average, 335 correct gene activations out of a total of 384 genes). Again, the benefit of increasing the size of the regulatory layer appeared to diminish beyond eight regulatory nodes.

Task B was in several respects a more difficult problem than Task A. Whereas fitness in the first task was measured solely on the basis of the end products of the differentiation process (the terminal cells), in the second task each of the cells was required to maintain a specified pattern of activation at every point 
in its trajectory. Task B was also more difficult due to the greater depth of the lineage and the increased density of the expression patterns. The pattern of each cell differed from that of its sibling by only one bit, resulting in many parts of the lineage tree having very similar expression patterns.

\subsection{Task C: Termination of Cell Lineages}

\subsubsection{Aim}

The final simulation task aimed to further investigate the ability of DRGNs to control temporal sequences of information and provide correct output signals at appropriate points in time. At a certain point in development, differentiation of a particular cell lineage is complete, and division ceases. The timing of this event is also under genetic control. One of the best understood examples of genes controlling developmental timing in C. elegans is the interaction between the lin-4 and lin-14 genes. These two genes control the timing of cell division events in multiple cell types. Mutations to these genes result in phenotypes in which specific stages of larval development are either skipped or repeated. The temporal pattern of larval development is controlled by the level of expression of lin-14, which decreases over time due to repression brought about by increasing levels of lin-4 activation. Other interactions of this type have since been discovered (for a review, see [30]).

\subsubsection{Task Description}

The final developmental task required the evolution of a network that was able to specify the appropriate terminal point of each lineage beyond which cells stopped dividing. As targets, we used several of the sublineages ${ }^{2}$ taken from cell lineage of $C$. elegans [36] (see Figure 4). Each of these sublineages forms a

\footnotetext{
${ }^{2}$ Note that, while the term 'sublineage' has been used to describe an intrinsically determined fragment of a lineage [36], we use it more generally to refer to the portion of a lineage consisting of all the descendants of a particular founder cell.
} 
branch of the lineage tree of initial cell divisions shown in Figure 2. One of the structural genes was designated to have control over the the termination of cell division, such that a cell divided so long as this gene was active, but stopped dividing once this gene became inactive. As in Task B, the network was required to match the correct decisions of all cells at a given level before the next level was generated.

\subsubsection{Method}

The network structure, error measures and EA were used as described in Section 4. Four different target lineages corresponding to the sublineages of cells D (39 terminal cells), E (39 terminal cells), C (95 terminal cells) and MS (187 terminal cells) were used. The effect of varying the level of regulation was explored by varying the size of the regulatory layer $\left(N_{r}=2,4,8,16\right)$. For each combination of target lineage and $N_{r}, 10$ trials were run, each initialised with a random set of network weights. Each trial was run for 250,000 generations, or until a solution with an NGE of zero was found.

\subsubsection{Results}

DRGNs were evolved that were able to generate sublineages $\mathrm{D}, \mathrm{E}$, and $\mathrm{C}$ with $100 \%$ accuracy and the MS sublineage with $66.2 \%$ accuracy (see Figure 7 ). The performance on each of the sublineages is discussed in order of increasing size.

Sublineage D: For each regulatory layer size, the EA was able to find a perfect solution in at least one of the ten trials and all ten of the trials involving networks with eight and sixteen regulatory genes found a perfect solution.

Sublineage E: Perfect solutions were found by the EA in all ten of the trials involving networks with sixteen regulatory nodes but only two of the trials involving networks with eight regulatory nodes. Despite the fact that sublineages 
$\mathrm{D}$ and $\mathrm{E}$ both had the same number of terminal cells (39), the asymmetrical arrangement of the left and right branches of sublineage $\mathrm{E}$ rendered it a more difficult task. For sublineage $\mathrm{D}$, the network was not required to make any symmetry breaking decision until the third division. For sublineage E, this decision was not only required one division earlier, but the subsequent trajectories were mutually exclusive, increasing the information needing to be stored in the regulatory interactions.

Sublineage C: The EA found perfect solutions in all but one of the trials involving networks with sixteen regulatory nodes, and in six of the ten trials involving networks with eight regulatory nodes.

Sublineage MS: The MS sublineage proved considerably more difficult than the other sublineages. The greatest accuracy $(66.2 \%)$ was obtained by a DRGN with sixteen regulatory nodes. As well as being more than twice as large as the previous sublineage, the MS sublineage also had a more irregular structure. The MS sublineage consists of two major branches (MSa and MSp) that are highly similar in many respects (as in sublineage C), however, there are two critical differences. The right branch contains a lineage that undergoes apoptosis (programmed cell death) after five divisions - a division earlier than the point at which the corresponding lineage on the left branch terminates. This irregularity posed a problem for the DRGNs as they were essentially being required to learn to recognise a single special case out of 32 otherwise regular lineages. Similarly, the left branch contained a single lineage that terminated after eight divisions, where the corresponding lineage in the right branch terminated after seven divisions, representing a single special case out of the 28 otherwise regular lineages that had not yet terminated at that point. The exact cause of these types of irregularities in the real C. elegans cell lineages varies on a case by case basis. However, one mechanism that has been identified is the competitive 
interaction between cells generated along the midline of the embryo [36]. The failure of DRGNs at this point suggests a possible case where a more information rich description of a cell's spatial position or other physical factors may be required.

\section{Discussion and Conclusion}

The results reported in this paper demonstrate that a network of interacting genes is able to control the developmental trajectories of a moderate number of individual cells with only a minimal level of external input. Networks with less than ten regulatory genes were consistently able to control the activity patterns of up to twice as many structural genes on tasks involving target patterns that were random (Task A), correlated (Task B) or consisted of complex symmetrical and asymmetrical lineages (Task C). The only information provided to the network in each case was its relative position in the lineage after division - whether it lay on the left or the right (anterior or posterior).

The first task, matching specified target patterns, showed that generating arbitrary patterns of coordinated behaviour is relatively easy for DRGNs. Furthermore, a trade was observed between the complexity of the task, represented by the size of the target patterns, and the size of search space, determined by the number of regulatory nodes in a network. This balance challenges the intuition that larger problems are necessarily going to be solved more efficiently by a larger network.

The second task, involving the generation of unique patterns for each level of a cell lineage in a combinatorial fashion, demonstrated the ability of DRGNs to maintain and transmit information over a number of division cycles. It also highlighted a possible limitation of the EA used to find the network weights. Theoretically, a network with $N$ structural genes should be able to represent $2^{N}$ unique patterns, suggesting that it is not the capacity of the network that 
limits performance, but rather the ability of the EA to find a suitable means of representing the trajectories. Examining the final evolved networks revealed that they tended to use most of their state space to represent the early divisions. While this resulted in very robust performance on the initial divisions, later divisions required the state space to be partitioned into increasingly small compartments, and errors became more frequent.

The third task, matching the lineage topologies of several C. elegans sublineages, demonstrated the ability of the DRGNs to store temporal information. Examining the state space trajectories representing each lineage revealed that the network exploited gradients of activity to control the timing of division termination. This final task also demonstrated that not all lineage topologies were equally easy to generate and that their difficulty was not necessarily correlated with the size of the lineage. It was significantly easier to find a network able to generate sublineage $\mathrm{D}$ than sublineage $\mathrm{E}$, despite the fact that both contained 39 cells. The irregularity of sublineage $\mathrm{E}$, with respect to its self similarity across different scales, proved to be more challenging for the networks. This insight was supported by further tests in which cell lineages were generated from random networks - a significant portion of the lineages contained self similar patterns, whereas none contained the type of asymmetric pattern observed in sublineage E.

The competence of the network performance suggests that the DRGN model is sufficient, given the definition of our developmental tasks, to simulate biologically inspired behaviours up to a reasonable level of complexity. The failures of the networks are also instructive, indicating a potential role for other informational signals in the control of embryonic development. The simplicity of the DRGN model makes it amenable to the use of machine learning techniques to find networks able to generate specific patterns of behaviour. Furthermore, this simplicity enables analysis of the found networks, providing some insight into how the target behaviours are being achieved. 
The innate tendency of control mechanisms based on recurrent networks to generate systematic or quasi-systematic structures may offer some insight into the topologies of cell lineages observed in nature. If the control of one particular lineage topology is more easily generated than another, it is possible that the patterns of development exhibited by biological organisms may reflect inherent biases in their gene network controllers. The fact that some of these self similar structures are broken, not by innate control of termination, but rather by apoptosis, suggests both a means for evolution to circumvent these innate control tendencies and also a possible explanation for some instances of apoptosis. It is not always clear why particular cell lineages are formed at all if the resulting cell is only going to be killed at some later point in development [26]. One answer may be that it is easier to internally program the development of a systematic lineage topology and utilise additional signals to "prune" excess branches. Incorporating the additional mechanisms required to explore this hypothesis represent one of the potential extensions that could be applied to the DRGN model.

The design of the DRGN model and its assessment using a variety of cell lineage tasks provide a window into the complexity of the control tasks solved by every multicellular organism as it develops from a single cell to an embryo. It is axiomatic that development involves the control of gene expression in both time and space, and simulation forces the computational aspects of these processes to be explicitly incorporated into models. In particular, simulating cell lineage control with the DRGN model enabled us to explore the influence of network architectures on the shape of developmental lineages. Observed cell lineage topologies may reflect inherent biases of the developmental processes that construct them, which are in turn constrained by the fundamental properties of the underlying gene networks. 


\section{Acknowledgments}

An early version of this paper appeared in the proceedings of ACAL 2003 [14] and we thank H. Abbass, the conference reviewers and two anonymous reviewers of this paper for their comments. We also thank R. Azevedo, J. Hallinan, J. Mattick, U. Platzer, B. Tonkes and members of the Complex and Intelligent Systems group for useful discussions and comments. This study was supported by an APA to NG and an ARC grant to JW.

\section{References}

1. Alberts, B., Bray, D., Lewis, J., Raff, M., Roberts, K., \& Watson, J. D. (1994). Molecular Biology of the Cell, 3rd ed. New York, NY: Garland.

2. Bäck, T., Fogel, D., \& Michalewicz, Z. (1997). Handbook of Evolutionary Computation. Oxford, UK: Oxford University Press.

3. P. J. Bentley (Ed.). (1999). Creative Evolutionary Design. San Francisco, CA: Morgan Kaufman.

4. Bodnar, J. W. (1997). Programming the Drosophila embryo. Journal of Theoretical Biology, 188, 391-445.

5. Bongard, J. \& Pfeifer, R. (2003). Evolving complete agents using artificial ontogeny. In F. Hara \& R. Pfeifer (Eds.), Morpho-functional Machines: The New Species (Designing Embodied Intelligence) (pp. 237-258). Berlin: Springer-Verlag.

6. Braun, V., Azevedo, R. B. R., Gumbel, M., Agapow, P.-M., Leroi, A. M. \& Meinzer, H.-P. (2003). ALES: cell lineage analysis and mapping of developmental events. Bioinformatics, 19, 851-858. 
7. Davidson, E. H., McClay, D. R., \& Hood, L. (2003). Regulatory gene networks and the properties of the developmental process. Proceedings of the National Academy of Science of the USA, 100, 1475-1480.

8. de Jong, H. (2002). Modeling and simulation of genetic regulatory systems: A literature review. Journal of Computational Biology, 9, 67-103.

9. Di Paolo, E. A., Noble, J., \& Bullock, S. (2000). Simulation models as opaque thought experiments. In M. Bedau, J. McCaskill, N. Packard, \& S. Rasmussen (Eds.), Artificial Life VII (pp. 497-506). Cambridge, MA: The MIT Press/Bradford Books.

10. Eggenberger, P. (2003). Genome-physics interaction as a new concept to reduce the number of genetic parameters in artificial evolution. In R. Sarker, R. Reynolds, H. Abbass, K.-C. Tan, R. McKay, D. Essam, \& T. Gedeon (Eds.), Proceedings of the IEEE 2003 Congress on Evolutionary Computation (pp. 191-198). Piscataway, NJ: IEEE Press.

11. Elman, J. L. (1990). Finding structure in time. Cognitive Science, 14, $179-211$.

12. Fleischer, K. (1996). Investigations with a multicellular developmental model. In C. G. Langton \& T. Shimohara (Eds.), Artificial Life V (pp. 229-236). Cambridge, MA: The MIT Press/Bradford Books.

13. Furusawa, C. \& Kaneko, K. (2000). Complex organisation in multicellularity as a necessity in evolution. Artificial Life, 6, 265-281.

14. Geard, N. \& Wiles, N. (2003). A gene regulatory network for cell differentiation in Caenorhabditis elegans. In H. Abbass \& J. Wiles, Proceedings of the Australian Conference on Artificial Life (ACAL 2003) (pp. 86-100) Canberra, Australia: UNSW. 
15. Hasty, J., McMillen, D., Isaacs, F., \& Collins, J. J. (2001). Computational studies of gene regulatory networks: in numero molecular biology. Nature Reviews Genetics, 2, 268-279.

16. Hogeweg, P. (2000). Shapes in the shadow: Evolutionary dynamics of morphogenesis. Artificial Life, 6, 85-101.

17. Hornby, G. S., Lipson, H., \& Pollack, J. B. (2003). Generative representations for the automated design of modular physical robots. IEEE Transactions on Robotics and Automation, 19, 703-719.

18. Huang, S. \& Ingber, D. E. (2000). Shape-dependent control of cell growth, differentiation, and apoptosis: Switching between attractors in cell regulatory networks. Experimental Cell Research, 261, 91-103.

19. Jan, N. J. \& Jan, L. Y. (1998). Asymmetric cell division. Nature, 392, $775-778$.

20. Kaletta, T., Schnabel, H. \& Schnabel, R. (1997). Binary specification of the embryonic lineage in Caenorhabditis elegans. Nature, 390, 294-298.

21. Kauffman, S. A. (1993). The Origins of Order: Self-Organization and Selection in Evolution. Oxford, UK: Oxford University Press.

22. Kitano, H., Hamahashi, S., \& Luke, S. (1998). The perfect C. elegans project: An initial report. Artificial Life, 4, 141-156.

23. Kumar, S. \& Bentley, P. (2003). Biologically inspired evolutionary design. In A. M. Tyrrell, P. C. Haddow, \& J. Torresen (Eds.), Proceedings of the International Conference on Evolvable Systems 2003 (pp. 57-68). Berlin: Springer.

24. Labouesse, M. \& Mango, S. E. (1999). Patterning the C. elegans embryo. Trends in Genetics, 15, 307-313. 
25. Lin, R., Hill, R. J., \& Priess, J. R. (1998). POP-1 and anterior-posterior fate decisions in C. elegans embryos. Cell, 92, 229-239.

26. Metzstein, M. M., Stanfield, G. M. \& Horvitz, H. R. (1998). Genetics of programmed cell death in C. elegans: past, present and future. Trends in Genetics, 14, 410-416.

27. Mjolsness, E., Sharp, D. H., \& Reinitz, J. (1991). A connectionist model of development. Journal of Theoretical Biology, 152, 429-453.

28. Orphanides, G. \& Reinberg, D. (2002). A unified theory of gene expression. Cell, 108, 439-451.

29. Rose, L. S. \& Kemphues, K. J. (1998). Early patterning of the C. elegans embryo. Annual Review of Genetics, 32, 521-545.

30. Rougvie, A. E. (2001). Control of developmental timing in animals. Nature Reviews Genetics, 2, 690-701.

31. Schnabel, R., Hutter, H., Moerman, D. \& Schnabel, H. (1997). Assessing normal embryogenesis in Caenorhabditis elegans using a 4D microscope: variability of development and regional specification. Developmental Biology, 184, 234-265.

32. Sipper, M. (2002). Machine Nature: The Coming Age of Bio-Inspired Computing. New York, NY: McGraw Hill.

33. Solé, R. V., Salazar-Ciudad, I., \& Garcia-Fernández, J. (2002). Common pattern formation, modularity and phase transitions in a gene network model of morphogenesis. Physica A, 305, 640-654.

34. Somogyi, R. \& Sniegoski, C. A. (1996). Modelling the complexity of genetic networks: Understanding multigenic and pleiotropic regulation. Complexity, $1,45-63$. 
35. Stanley, K. O. \& Miikkulainen, R. (2003). A taxonomy for artificial embryogeny. Artificial Life, 9, 93-130.

36. Sulston, J. E., Schierenberg, E., White, J. G., \& Thompson, J. N. (1983). The embryonic cell lineage of the nematode Caenorhabditis elegans. Developmental Biology, 100, 64-119.

37. Vohradský, J. (2001). Neural model of the genetic network. The Journal of Biological Chemistry, 276, 36168-36173.

38. Wolpert, L. (1998). The Principles of Development. Oxford, UK: Oxford University Press.

39. Wuensche, A. (1998). Genomic regulation modeled as a network with basins of attraction. In R. B. Altman, A. K. Dunker, L. Hunter, \& T. E. Klein (Eds.), Pacific Symposium on Biocomputing '98 (pp. 89-102). Singapore: World Scientific. 


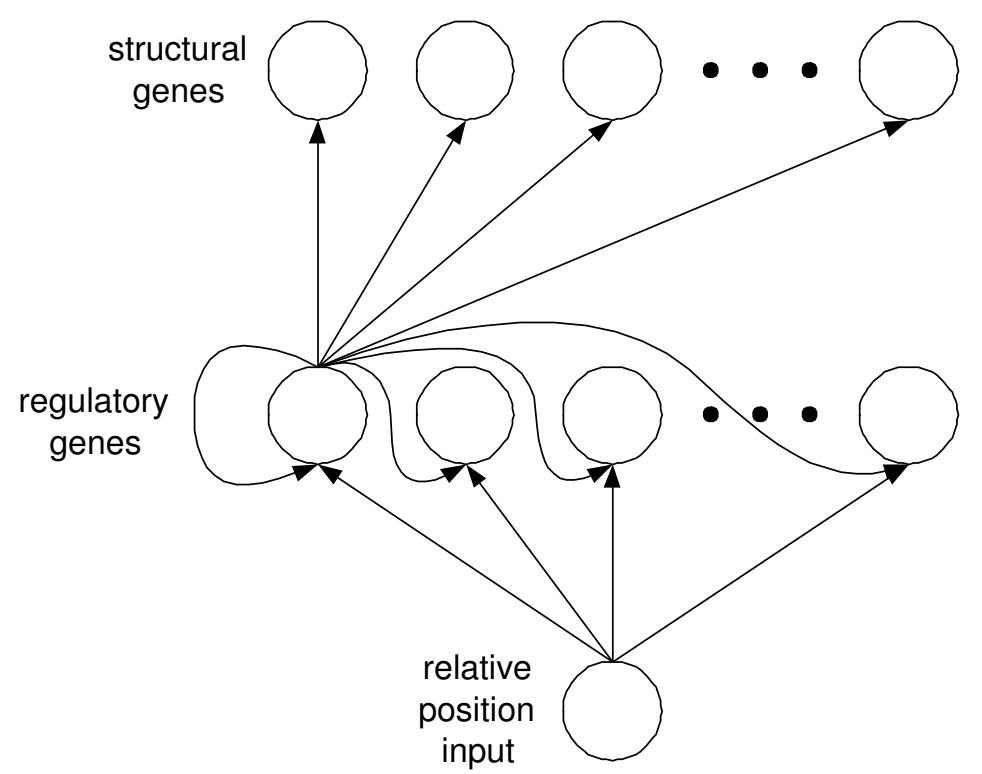

Figure 1: The structure of the DRGN model. Gene regulation is modelled using a partially connected network of $N$ nodes. Section 4.1 describes the different types of gene and the justification for the network structure. 


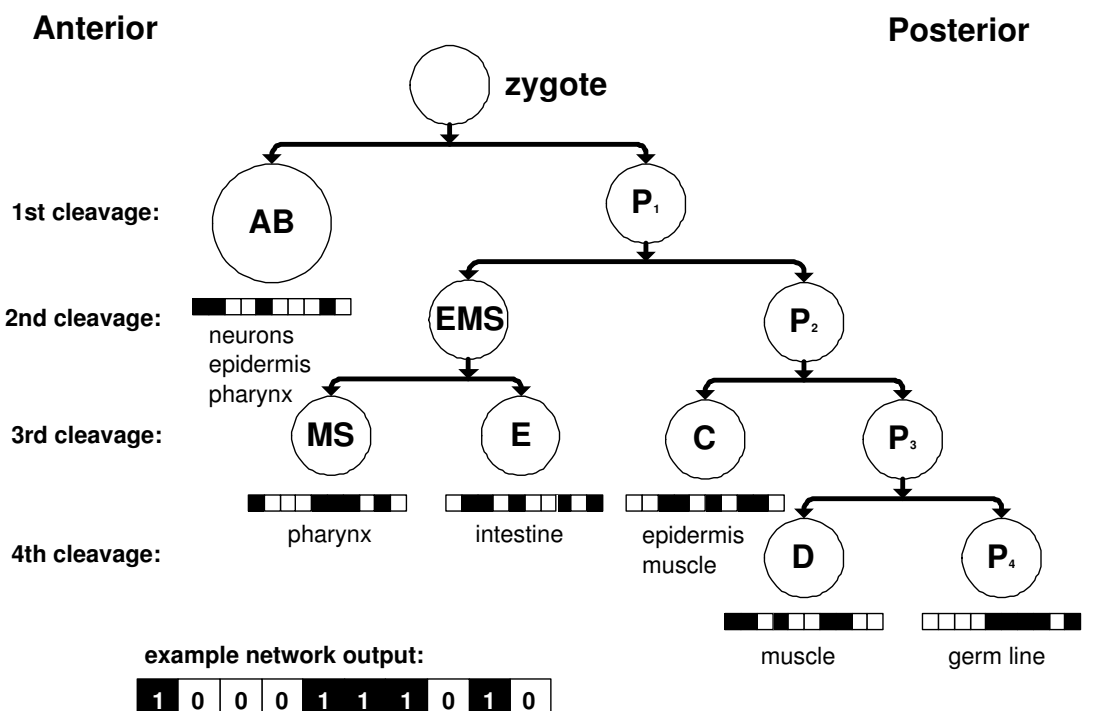

Figure 2: The cell lineage of very early C. elegans embryogenesis. Each precursor cell cleavage results in the production of one somatic cell, which will divide to generate epidermal, intestinal, neural and muscle cells, and a further precursor cell. The final precursor cell, $\mathrm{P}_{4}$, gives rise to the germ line (redrawn from [36]). Also shown is an example of the target activation patterns used in Task A, for a trial with 10 structural genes. The enlarged example pattern illustrates the correspondence between the patterns in the figure and the target activation of the structural genes in the simulations. 


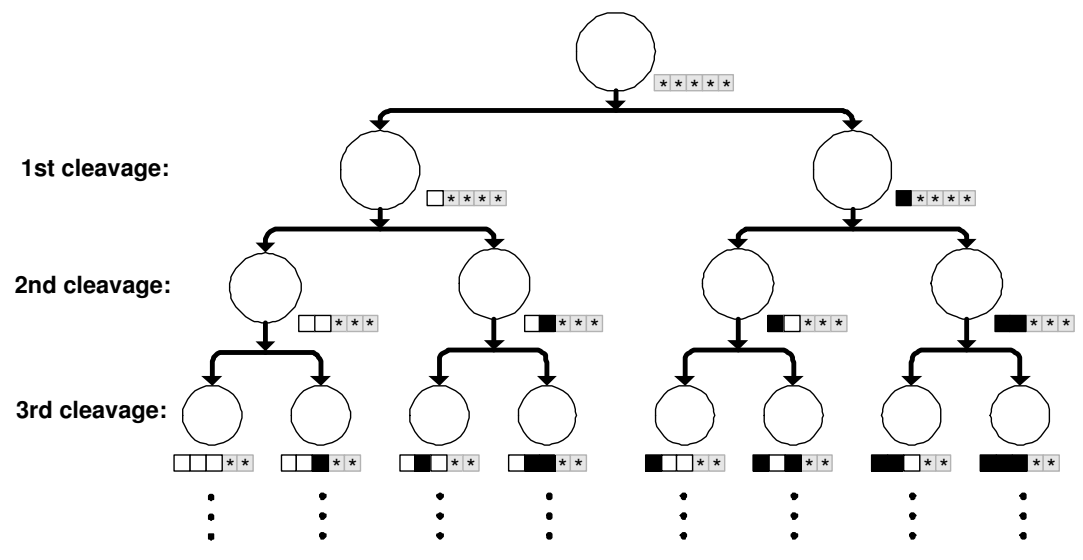

Figure 3: A cell lineage detailing the production of unique cell lineages by combinatorial gene activation. The target patterns of gene activation used in Task B are shown below each cell. The representation is the same as that shown in Figure 2 except that greyed out squares represent genes whose output is not defined at that level of division. 

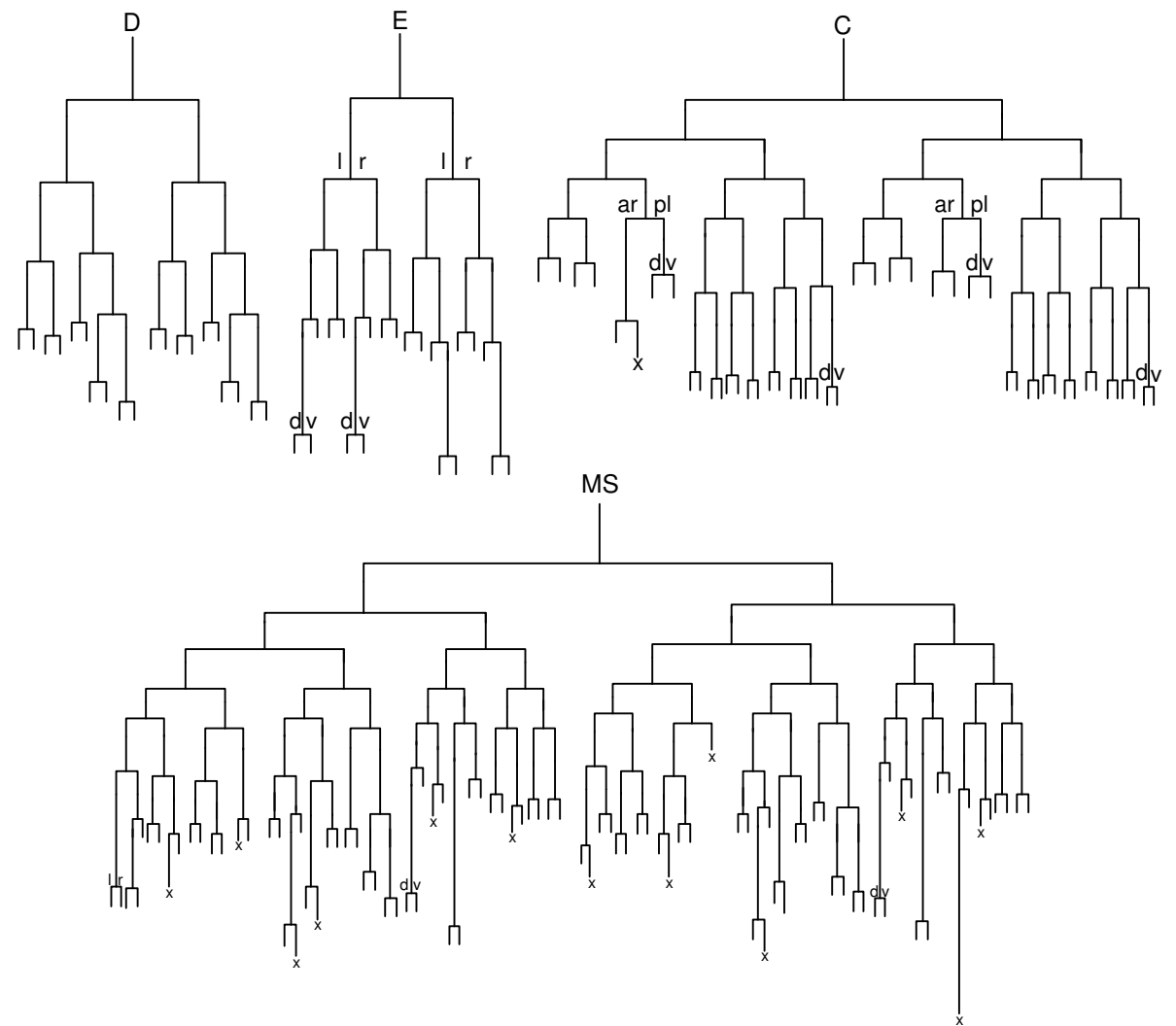

Figure 4: The C. elegans sublineages used in Task C. All divisions occur along the anterior-posterior axis unless otherwise specified as being along the dorsal(d)-ventral(v) or left(l)-right(r) axes. An x indicates a cell that undergoes apoptosis. The vertical axis of each lineage provides an indication of the relative timing of each division event, however, for the purposes of the simulations reported here, all divisions at a given level occurred simultaneously. Redrawn from [36]. 


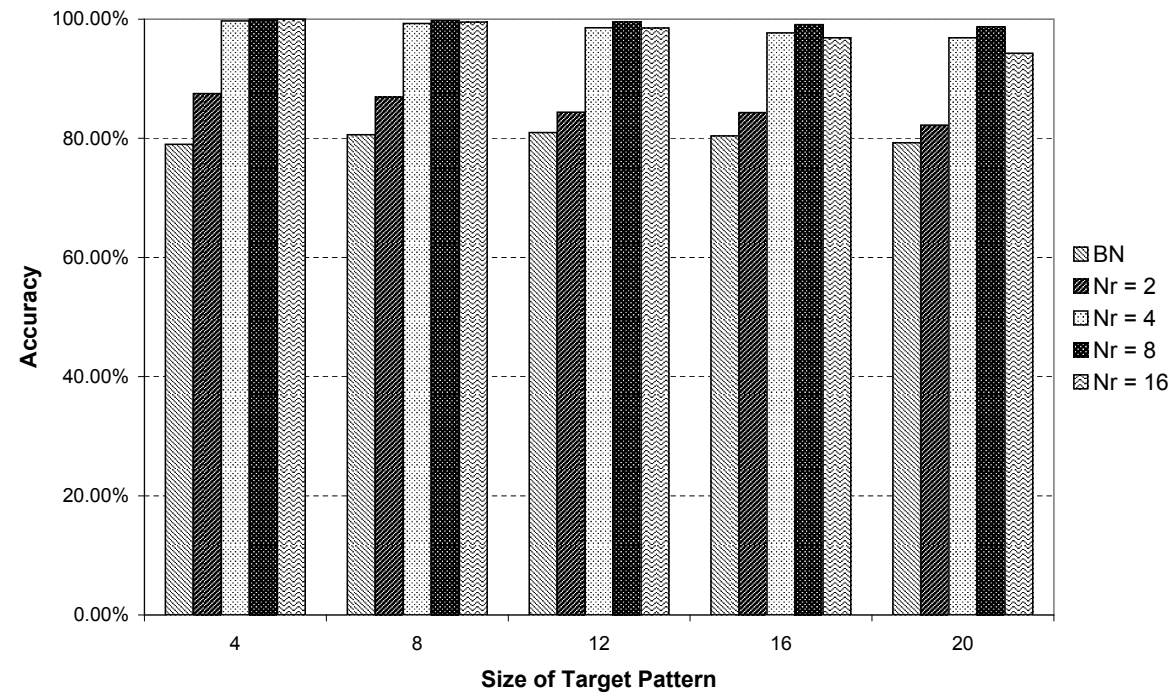

Figure 5a: The DRGN performance on Task A. All results are averaged over 100 trials (10 random starting positions for each of 10 random sets of target patterns). For comparison the accuracy of the best performing Boolean network (with $N_{r}=16$ and $\left.K=2\right)(\mathrm{BN})$ is also shown. Accuracy was defined by the number of remaining incorrect structural genes (NGE) as a percentage of the total number of target genes after 50,000 generations. 


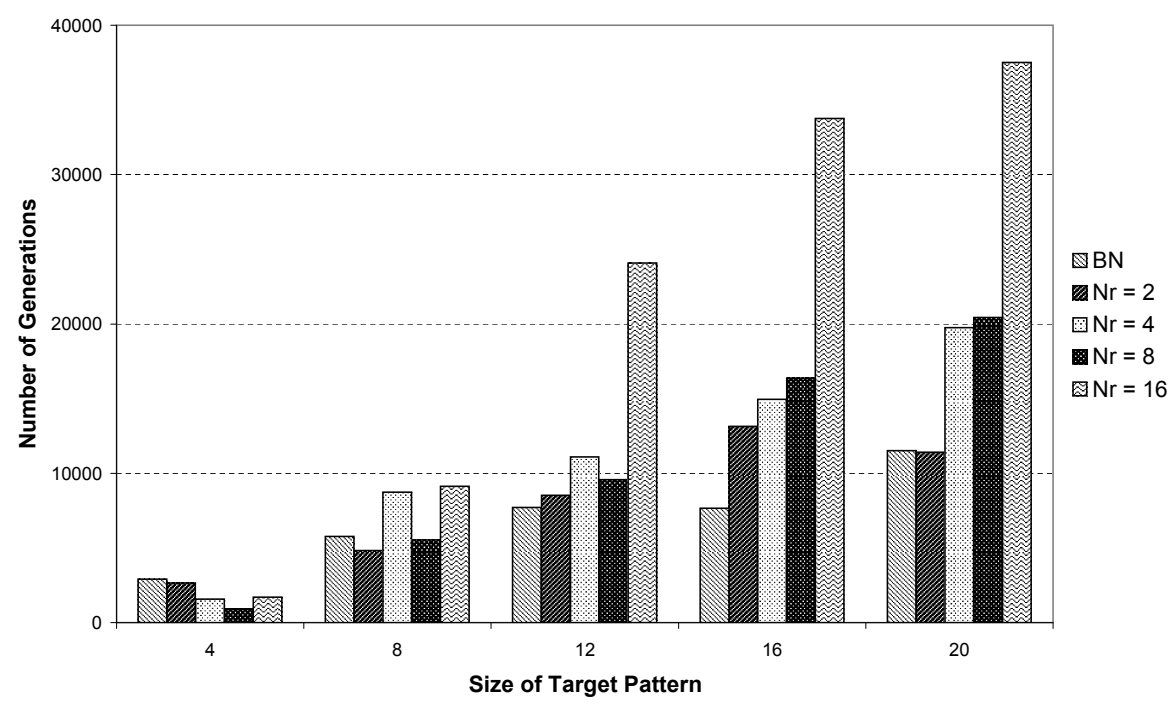

Figure 5b: The length of time taken by the EA in Task A before no further improvement was found, averaged over ten trials. 


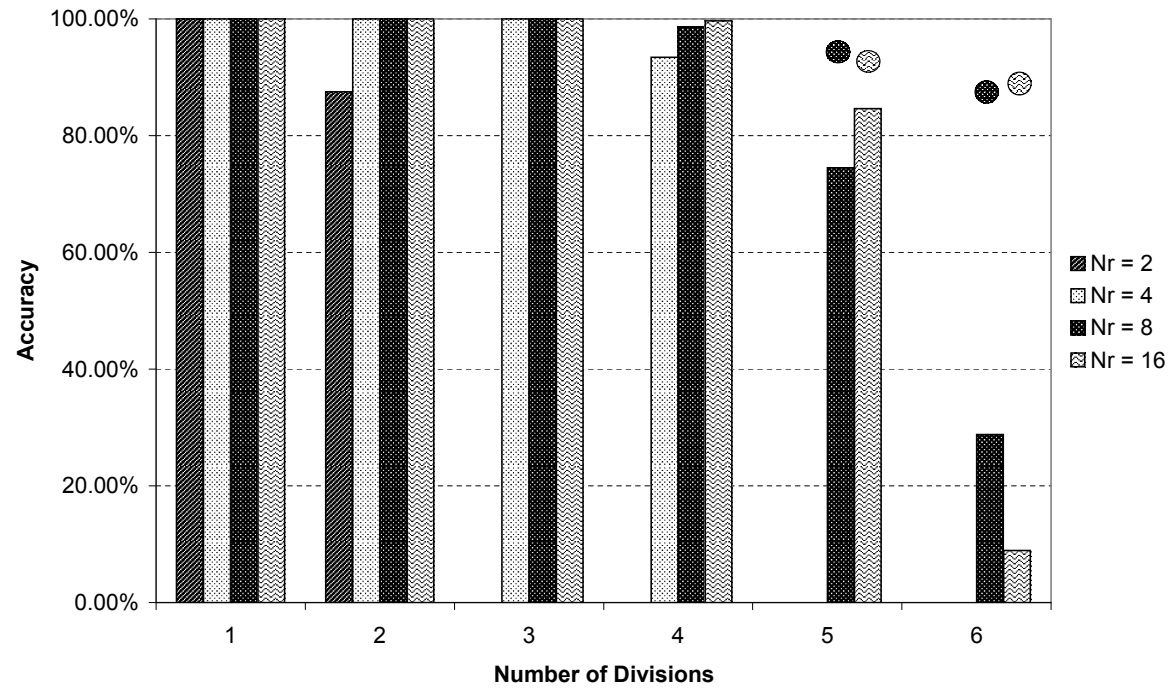

Figure 6: The DRGN performance on Task B. The average accuracy of the DRGNs is shown for each level of division, after there had been no improvement for 50,000 generations. Each bar indicates the accuracy averaged across all ten trials. Average accuracy after the second division for $N_{r}=2$ and after the fourth division for $N_{r}=4$ was $0.00 \%$. The circles indicate the accuracy averaged across only those trials that actually reached that level of division (i.e. the trials in which a network correctly specified only four levels of division were not included for the calculation of accuracy at level five). 


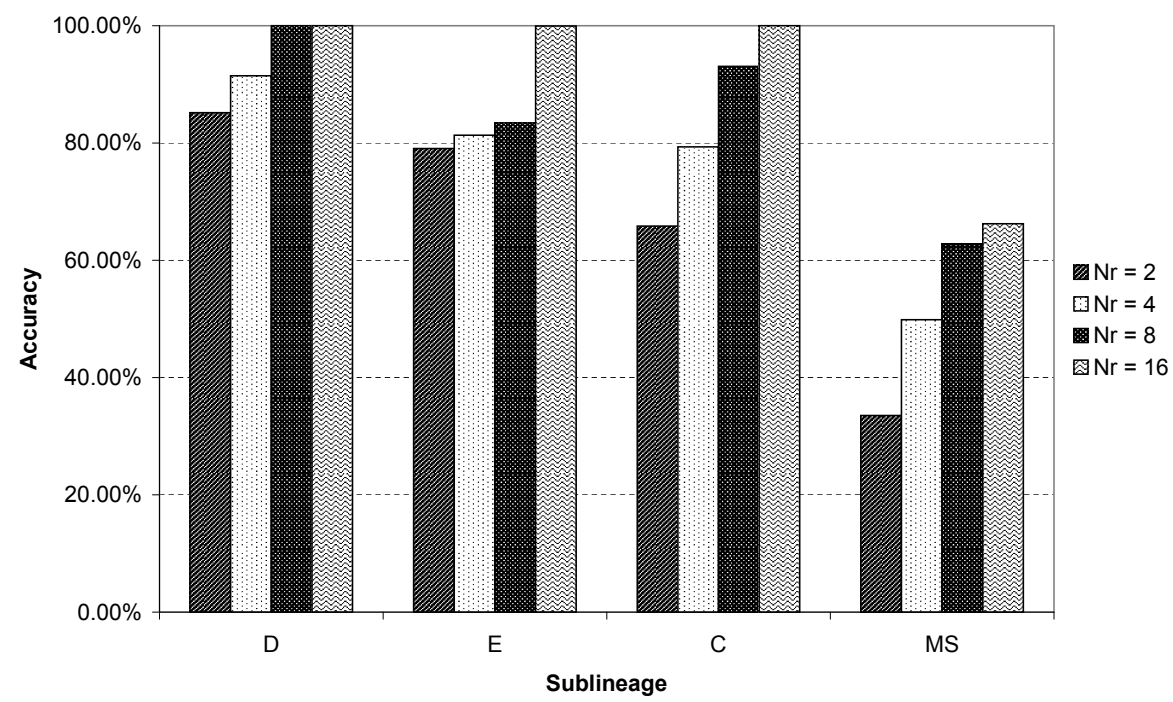

Figure 7: The DRGN performance on Task C. The average accuracy achieved by the DRGNs on each of the D, E, C and MS sublineages of C. elegans, averaged over ten trials. 